\title{
An evolutionary formalism for weak quantum measurements
}

\author{
Apoorva Patel* and Parveen Kumar \\ Centre for High Energy Physics, Indian Institute of Science, Bengaluru 560 012, India
}

Unitary evolution and projective measurement are fundamental axioms of quantum mechanics. Even though projective measurement yields one of the eigenstates of the measured operator as the outcome, there is no theory that predicts which eigenstate will be observed in which experimental run. There exists only an ensemble description, which predicts probabilities of various outcomes over many experimental runs. We propose a dynamical evolution equation for the projective collapse of the quantum state in individual experimental runs, which is consistent with the well-established framework of quantum mechanics. In case of gradual weak measurements, its predictions for ensemble evolution are different from those of the Born rule. It is an open question whether or not suitably designed experiments can observe this alternate evolution.

Keywords: Born rule, decoherence, density matrix, fixed point, quantum trajectory, state collapse.

\section{The problem}

THIS talk is about filling a gap in the existing framework of quantum mechanics. At its heart, quantum mechanics contains two distinct dynamical rules for evolving a state. One is unitary evolution, specified by the Schrödinger equation

$$
i \frac{\mathrm{d}}{\mathrm{d} t}|\psi\rangle=H|\psi\rangle, \quad i \frac{\mathrm{d}}{\mathrm{d} t} \rho=[H, \rho] .
$$

It is continuous, reversible and deterministic. The other is the von Neumann projective measurement, which gives one of the eigenvalues of the measured observable as the measurement outcome and collapses the state to the corresponding eigenvector. With $P_{i}$ denoting the projection operator for the eigenvalue $\lambda_{i}$,

$$
\left.|\psi\rangle \rightarrow P_{i}|\psi\rangle /\left|P_{i}\right| \psi\right\rangle \mid, P_{i}^{2}=P_{i}=P_{i}^{\dagger}, \sum_{i} P_{i}=I .
$$

\footnotetext{
*For correspondence. (e-mail: adpatel@cts.iisc.ernet.in)
}

This change is discontinuous, irreversible and probabilistic in the choice of ' $i$ '. It is consistent on repetition, i.e. a second measurement of the same observable on the same system gives the same result as the first one.

Both these evolutions, not withstanding their dissimilar properties, take pure states to pure states. They have been experimentally verified so well that they are accepted as axioms in the standard formulation of quantum mechanics. Nonetheless, the formulation misses something: While the set of projection operators $\left\{P_{i}\right\}$ is fixed by the measured observable, only one ' $i$ ' occurs in a particular experimental run, and there is no prediction for which ' $i$ ' that would be.

What appears instead in the formulation is the probabilistic Born rule, requiring an ensemble interpretation for verification. Measurement of an observable on a collection of identically prepared quantum states gives

$$
\operatorname{prob}(i)=\left\langle\psi\left|P_{i}\right| \psi\right\rangle=\operatorname{Tr}\left(P_{i} \rho\right), \rho \rightarrow \sum_{i} P_{i} \rho P_{i}
$$

This rule evolves pure states to mixed states. All predicted quantities are expectation values obtained as averages over many experimental runs. The appearance of a mixed state also necessitates a density matrix description, instead of a ray in the Hilbert space description for a pure state.

Over the years, many attempts have been made to combine these two distinct quantum evolution rules in a single framework. Although the problem of which ' $i$ ' will occur in which experimental run has remained unsolved, progress has been achieved in understanding the 'ensemble evolution' of a quantum system.

\section{Environmental decoherence}

The system, the measuring apparatus as well as the environment - all are ultimately made from the same set of fundamental building blocks. With quantum theory successfully describing the dynamical evolution of all the fundamental blocks, it is logical to consider the proposition that the whole universe is governed by the same set of basic quantum rules. The essential difference between the system and the environment is that the degrees of freedom of the system are observed while those of the environment are not. (In a coarse-grained view, 
unobserved degrees of freedom of the system can be treated in the same manner as those of the environment.) All the unobserved degrees of freedom then need to be 'summed over' to determine how the remaining observed degrees of freedom evolve.

No physical system is perfectly isolated from its surroundings. Interactions between the two, with a unitary evolution for the whole universe, entangles the observed degrees of freedom of the system with the unobserved degrees of freedom of the environment. When the unobserved degrees of freedom are summed over, a pure but entangled state for the universe reduces to a mixed state for the system

$$
|\psi\rangle_{\mathrm{SE}} \rightarrow U_{\mathrm{SE}} \operatorname{Tr}|\psi\rangle_{\mathrm{SE}}, \rho_{\mathrm{S}}=\operatorname{Tr}_{\mathrm{E}}\left(\rho_{\mathrm{SE}}\right), \rho_{\mathrm{S}}^{2} \neq \rho_{\mathrm{S}}
$$

In general, the evolution of a reduced density matrix is linear, Hermiticity preserving, trace preserving and positive, but not unitary. Using a complete basis for the environment $\left\{|\mu\rangle_{\mathrm{E}}\right\}$, such a superoperator evolution can be expressed in the Kraus decomposition form

$$
\begin{aligned}
& \rho_{S} \rightarrow \sum_{\mu} M_{\mu} \rho_{S} M_{\mu}^{\dagger}, \\
& M_{\mu}={ }_{\mathrm{E}}\left\langle\mu\left|U_{\mathrm{SE}}\right| 0\right\rangle_{\mathrm{E}}, \sum_{\mu} M_{\mu}^{\dagger} M_{\mu}=I .
\end{aligned}
$$

This description explains the probabilistic ensemble evolution of quantum mechanics in a language similar to that of classical statistical mechanics. But it still has no mechanism to explain the projective collapse of a quantum state. (Ensemble averaging is often exchanged for ergodic time averaging in equilibrium statistical mechanics, but that option is not available in unitary quantum mechanics.)

Generically the environment has a much larger number of degrees of freedom than the system. Then, in the Markovian approximation which assumes that information leaked from the system does not return, the evolution of the reduced density matrix can be converted to a differential equation. With the expansion

$$
M_{0}=I+\mathrm{d} t(-i H+K)+\ldots, M_{\mu \neq 0}=\sqrt{\mathrm{d} t} L_{\mu}+\ldots
$$

eq. (5) leads to the Lindblad master equation ${ }^{1,2}$

$$
\begin{aligned}
& \frac{\mathrm{d}}{\mathrm{d} t} \rho=i[\rho, H]+\sum_{\mu} \mathcal{L}\left[L_{\mu}\right] \rho, \\
& \mathcal{L}\left[L_{\mu}\right] \rho=L_{\mu} \rho L_{\mu}^{\dagger}-\frac{1}{2} \rho L_{\mu}^{\dagger} L_{\mu}-\frac{1}{2} L_{\mu}^{\dagger} L_{\mu} \rho .
\end{aligned}
$$

The terms on the r.h.s. involving sum over $\mu$ modify the unitary Schrödinger evolution, while $\operatorname{Tr}(\mathrm{d} \rho / \mathrm{d} t)=0$ preserves the total probability. When $H=0$, the fixed point of the evolution is a diagonal $\rho$, in the basis that diagonalizes $\left\{L_{\mu}\right\}$. This preferred basis is determined by the system-environment interaction. (When there is no diagonal basis for $\left\{L_{\mu}\right\}$, the evolution leads to equipartition, i.e. $\rho \propto I$.) Furthermore, the off-diagonal components of $\rho$ decay due to destructive interference among environmental contributions with varying phases, which is known as decoherence.

This modification in the evolution of a quantum system, due to its coupling to unobserved environmental degrees of freedom provides the correct ensemble interpretation and a quantitative understanding of how the off-diagonal components of $\rho$ decay $^{3,4}$. Still the quantum theory is incomplete and we need to look further to solve the 'measurement problem' till it can predict the outcome of a particular experimental run.

\section{Going beyond}

A wide variety of theoretical approaches have been proposed to get around the quantum measurement problem. Some of them are physical, e.g. introduction of hidden variables with novel dynamics, and breakdown of quantum rules due to gravitational interactions. Some others are philosophical, e.g. questioning what is real and what is observable, in principle as well as by human beings with limited capacity. Given the tremendous success of quantum theory, realized with a 'shut up and calculate' attitude, and the stringent constraints that follow, none of the theoretical approaches have progressed to the level where they can be connected to readily verifiable experimental consequences.

Perhaps the least intrusive of these approaches is the 'many worlds interpretation". It amounts to assigning a distinct world (i.e. an evolutionary branch) to each probabilistic outcome, while we only observe the outcome corresponding to the world we live in. (It is amusing to note that this discussion meeting (held at IISc, 22-24 October 2014) is being held in a place where the slogan of the Department of Tourism is 'One state, Many worlds ${ }^{6}$.) Such an entanglement between the measurement outcomes and the observers does not violate any quantum principle, although the uncountable proliferation of evolutionary branches it supposes is highly ungainly. Truly speaking, the many worlds interpretation bypasses the measurement problem instead of solving it.

With the technological progress in making quantum devices, we need a solution to the measurement problem, not only for formal theoretical reasons, but also for increasing accuracy of quantum control and feedback ${ }^{4}$. A practical situation is that of the weak measurement ${ }^{7}$, typically realized using a weak system-apparatus coupling, 
where information about the measured observable is extracted from the system at a slow rate. Such a stretching out of the timescale allows one to monitor how the system state collapses to an eigenstate of the measured observable, and to track properties of the intermediate states created along the way by an incomplete measurement. Knowledge of what really happens in a particular experimental run (and not the ensemble average) would be invaluable in making quantum devices more efficient and stable.

\section{A way out}

Let us assume that the projective measurement results from a continuous geodesic evolution of the initial quantum state to an eigenstate $|i\rangle$ of the measured observable

$$
\left.|\psi\rangle \rightarrow Q_{i}(s)|\psi\rangle /\left|Q_{i}(s)\right| \psi\right\rangle \mid, Q_{i}(s)=(1-s) I+s P_{i},
$$

where the dimensionless parameter $s \in[0,1]$ represents the 'measurement time'. The density matrix then evolves as, maintaining $\operatorname{Tr}(\rho)=1$,

$$
\rho \rightarrow \frac{(1-s)^{2} \rho+s(1-s)\left(\rho P_{i}+P_{i} \rho\right)+s^{2} P_{i} \rho P_{i}}{(1-s)^{2}+\left(2 s-s^{2}\right) \operatorname{Tr}\left(P_{i} \rho\right)}
$$

Expansion around $s=0$ yields the differential equation

$$
\frac{\mathrm{d}}{\mathrm{d} s} \rho=\rho P_{i}+P_{i} \rho-2 \rho \operatorname{Tr}\left(P_{i} \rho\right)=\left\{\rho, P_{i}\right\}-\rho \operatorname{Tr}\left(\left\{\rho, P_{i}\right\}\right) .
$$

This simple equation describing an individual quantum trajectory has several remarkable properties. We can explore them, putting aside the argument that led to the equation. Explicitly,

- In addition to maintaining $\operatorname{Tr}(\rho)=1$, the nonlinear evolution preserves pure states. $\rho^{2}=\rho$ implies $\rho P_{i} \rho=$ $\rho \operatorname{Tr}\left(P_{i} \rho\right)$, and then

$\frac{\mathrm{d}}{\mathrm{d} s}\left(\rho^{2}-\rho\right)=\rho \frac{\mathrm{d}}{\mathrm{d} s} \rho+\left(\frac{\mathrm{d}}{\mathrm{d} s} \rho\right) \rho-\frac{\mathrm{d}}{\mathrm{d} s} \rho=0$.

For pure states, with $\langle\psi|(\mathrm{d} / \mathrm{d} s)| \psi\rangle=0$, we can also write $(\mathrm{d} / \mathrm{d} s)|\psi\rangle=\left(P_{i}-\left\langle\psi\left|P_{i}\right| \psi\right\rangle\right)|\psi\rangle$. So the component of the state along $P_{i}$ grows at the expense of the other orthogonal components.

- Each projective measurement outcome is the fixed point of the deterministic evolution

$$
\frac{\mathrm{d}}{\mathrm{d} s} \rho=0 \quad \text { at } \quad \rho_{i}^{*}=P_{i} \rho P_{i} / \operatorname{Tr}\left(P_{i} \rho\right)
$$

The fixed point nature of the evolution makes the measurement consistent on repetition. Note that onedimensional projections satisfy $P_{i} \rho P_{i}=P_{i} \operatorname{Tr}\left(P_{i} \rho\right)$.

- In a bipartite setting, the complete set of projection operators can be labelled as $\left\{P_{i}\right\}=\left\{P_{i_{1}} \otimes P_{i_{2}}\right\}$, with $\sum_{i} P_{i}=I$. Since the evolution is linear in the projection operators, a partial trace over the unobserved degrees of freedom produces the same equation (and hence the same fixed point) for the reduced density matrix for the system. Purification is thus a consequence of the evolution; for example, a qubit state in the interior of the Bloch sphere evolves to the fixed point on its surface.

- At the start of measurement, we expect the parameter $s$ to be proportional to the system-apparatus interaction, $s \sim\left\|H_{\mathrm{SA}}\right\| t$. To understand the approach towards the fixed point, let $\tilde{\rho} \equiv \rho-P_{i}$ for a one-dimensional projection, which satisfies

$\frac{\mathrm{d}}{\mathrm{d} s} \tilde{\rho}=\tilde{\rho} P_{i}+P_{i} \tilde{\rho}-2 \tilde{\rho}-2 \rho \operatorname{Tr}\left(P_{i} \tilde{\rho}\right)$

It follows that towards the end of measurement $s \rightarrow \infty$, and convergence to the fixed point is exponential with $\|\tilde{\rho}\| \sim \mathrm{e}^{-2 s}$, similar to the charging of a capacitor.

These properties make eq. (10) a legitimate candidate for describing the collapse of a quantum state during projective measurement. It represents a superoperator that preserves Hermiticity, trace and positivity, but is nonlinear. Because of its non-stochastic nature, it can model the single quantum trajectory specific to a particular experimental run.

Although eq. (10) does fill a gap in solving the measurement problem, with the preferred basis $\left\{P_{i}\right\}$ fixed by the system-apparatus interaction, we still need a separate criterion to determine which $P_{i}$ will occur in a particular experimental run. This is a situation reminiscent of spontaneous symmetry breaking ${ }^{8}$, where a small external field (with a smooth limit to zero) picks the direction, and the evolution is unique given that direction (stability of the direction depends on the thermodynamic size of the system). We do not have a prescription for such a choice, also referred to as a 'quantum jump'. The stochastic ensemble interpretation of quantum measurements is reproduced, according to the Born rule, when a particular $P_{i}$ is picked with probability $\operatorname{Tr}\left(P_{i} \rho(s=0)\right)$.

\section{Combining trajectories}

The probabilistic Born rule for measurement outcomes, eq. (3), is rather peculiar despite being tremendously successful. The reason is that the probabilities are determined by the initial state $\rho(s=0)$ and do not depend on 
the subsequent evolution of the state $\rho(s \neq 0)$. Any attempt to describe projective measurement as continuous evolution would run into the problem that the system would have to remember its state at the instant the measurement started until the measurement is complete. This is a severe constraint for any theory of weak measurement, where the measurement timescale is stretched out, and we can rightfully question whether the Born rule would hold in such a case.

It is possible to reconcile the Born rule with continuous projective measurements, by invoking retardation effects arising from special relativity for the speed of information travel between the system and the apparatus. Then the Born rule will be followed by sudden impulsive measurements with a duration shorter than the retardation time, but it may be violated by gradual weak measurements with a duration longer than the retardation time. We look beyond the Born rule satisfying possibility that the evolution trajectory corresponding to $P_{i}$ is chosen at the start of the measurement and remains unaltered thereafter, in order to look for more general evolutionary choices that may be suitable for weak measurements.

Let $w_{i}$ be the probability weight of the evolution trajectory for $P_{i}$, with $w_{i} \geq 0$ and $\sum_{i} w_{i}=1$. We have $w_{i}(s=0)=$ $\rho_{i i}(s=0)$ in accordance with the Born rule, while $w_{i}(s \neq 0)$ are some functions of $\rho(s)$. Then the trajectory averaged evolution of the density matrix during measurement is given by

$$
\frac{\mathrm{d}}{\mathrm{d} s} \rho=\sum_{i} w_{i}\left[\rho P_{i}+P_{i} \rho-2 \rho \operatorname{Tr}\left(P_{i} \rho\right)\right]
$$

It still preserves pure states, as per eq. (11). In terms of a complete set of projection operators, we can decompose $\rho=\sum_{j k} P_{j} \rho P_{k}$. The projected components evolve as

$$
\frac{\mathrm{d}}{\mathrm{d} s}\left(P_{j} \rho P_{k}\right)=P_{j} \rho P_{k}\left(w_{j}+w_{k}-2 \sum_{i} w_{i} \operatorname{Tr}\left(P_{i} \rho\right)\right) .
$$

This evolution obeys the identity, independent of the choice of $\left\{w_{i}\right\}$,

$$
\begin{aligned}
\frac{2}{P_{j} \rho P_{k}} \frac{\mathrm{d}}{\mathrm{d} s}\left(P_{j} \rho P_{k}\right)= & \frac{1}{P_{j} \rho P_{j}} \frac{\mathrm{d}}{\mathrm{d} s}\left(P_{j} \rho P_{j}\right) \\
& +\frac{1}{P_{k} \rho P_{k}} \frac{\mathrm{d}}{\mathrm{d} s}\left(P_{k} \rho P_{k}\right),
\end{aligned}
$$

with the consequence that the diagonal projections of $\rho$ completely determine the evolution of all the off-diagonal projections. The diagonal projections are all nonnegative. For one-dimensional projections, $P_{j} \rho(s) P_{j}=$ $d_{j}(s) P_{j}$ with $d_{j} \geq 0$, and we obtain

$$
P_{j} \rho(s) P_{k}=P_{j} \rho(0) P_{k}\left[\frac{d_{j}(s) d_{k}(s)}{d_{j}(0) d_{k}(0)}\right]^{1 / 2} .
$$

In particular, phases of the off-diagonal projections $P_{j} \rho P_{k}$ do not evolve, in sharp contrast to what happens during decoherence. Also, their asymptotic values, i.e. $P_{j} \rho(s \rightarrow \infty) P_{k}$, may not vanish whenever more than one diagonal $P_{j} \rho(s \rightarrow \infty) P_{j}$ remain nonzero. In a sense, decohering measurements select the Cartesian components of the quantum state in the eigenbasis provided by the system-apparatus interaction and lose information about the angular coordinates, while the collapse equation selects the radial components of the quantum state around the measurement fixed points leaving the angular components unchanged. Mathematically speaking, both measurement schemes are consistent.

It is easily seen that when all the $w_{i}$ are equal, no information is extracted from the system by the measurement and $\rho$ does not evolve. More generally, the diagonal projections evolve according to

$$
\frac{\mathrm{d}}{\mathrm{d} s} d_{j}=2 d_{j}\left(w_{j}-\sum_{i} w_{i} d_{i}\right)=2 d_{j}\left(\sum_{i \neq j}\left(w_{j}-w_{i}\right) d_{i}\right) \text {. }
$$

Here, with $\sum_{i} d_{i}=1, \sum_{i} w_{i} d_{i} \equiv \bar{w}$ is the weighted average of $\left\{w_{i}\right\}$. Clearly, the diagonal projections with $w_{j}>\bar{w}$ grow and the ones with $w_{j}<\bar{w}$ decay. Any $d_{j}$ that is zero initially does not change and the evolution is therefore restricted to the subspace spanned by all the $P_{j} \rho(s=0) P_{j} \neq 0$. Also, all the measured observable eigenstates, i.e. $\rho=P_{j}$ with $d_{j}=1$, are fixed points of the evolution. These features are stable under small perturbations of the density matrix.

Other fixed points of eq. (18) correspond to 'degenerate' situations where some of the $w_{j}$ (say $n>1$ in number) are equal and all the others vanish, i.e. $w_{j} \in\left\{0, \frac{1}{n}\right\}$. These fixed points are unstable under asymmetric perturbations that lift the degeneracy. It may be that other terms in the evolution Hamiltonian, which have been ignored throughout in our measurement description and whose contribution would have to be added to eq. (14) in describing complete evolution of the system, can stabilize them and make the evolution converge towards an $n$-dim degenerate subspace.

An appealing choice for the trajectory weights is the 'instantaneous Born rule', i.e. $w_{j}=w_{j}^{B} \equiv \operatorname{Tr}\left(P_{j} \rho(s)\right)$ throughout the measurement process. That avoids logical inconsistency in weak measurement scenarios, where one starts the measurement, pauses somewhere along the way, and then restarts the measurement. In this situation, the trajectory averaged evolution is

$$
\frac{\mathrm{d}}{\mathrm{d} s}\left(P_{j} \rho P_{k}\right)=P_{j} \rho P_{k}\left(w_{j}^{\mathrm{B}}+w_{k}^{\mathrm{B}}-2 \sum_{i}\left(w_{i}^{\mathrm{B}}\right)^{2}\right) .
$$


This evolution converges towards the $n$-dimensional subspace specified by the dominant diagonal projections of the initial $\rho(s=0)$. It is deterministic and does not follow eq. (3). The measurement result remains consistent under repetition though.

The evolution can be made stochastic, in a manner similar to the Langevin equation, by adding noise to the weights $w_{i}$ while still retaining $\sum_{i} w_{i}=1$. The weak measurement process is expected to contribute such a noise ${ }^{9-11}$. The resultant evolution and its dependence on the magnitude of the noise need to be investigated.

\section{Relation to the master equation}

The master equation is obtained assuming that the environmental degrees of freedom are not observed and hence are summed over. On the other hand, the degrees of freedom corresponding to the measured observable are observed in any measurement process with a definite outcome and cannot be summed over. In analysing the measurement process, we need to keep track of only those degrees of freedom of the apparatus that have a one-to-one correspondence with the system's eigenstates, and the rest can be kept aside. The crucial difference between the states of the system and the apparatus is that the system can be in a superposition of the eigenstates, but the apparatus has to end up in one of the pointer states only (and not their superposition).

In the traditional description, at the start of the measurement the joint state of the system and the apparatus can be chosen to be $\sum_{i} c_{i}|i\rangle_{S}|0\rangle_{\mathrm{A}}$, with $\sum_{i}\left|c_{i}\right|^{2}=1$. The system-apparatus interaction then unitarily evolves it to the entangled state $\Sigma_{i} c_{i}|i\rangle_{\mathrm{S}}|\tilde{i}\rangle_{\mathrm{A}}$. This evolution is a controlled unitary transformation (and not a copy operation). The preferred measurement basis is the Schmidt decomposition basis, ensuring a perfect correlation between the system eigenstate $|i\rangle_{\mathrm{S}}$ and the measurement pointer state $|\tilde{i}\rangle_{\mathrm{A}}$. In particular, the reduced density matrices of the system and the apparatus are identical at this stage. Thereafter, the state collapse picks one of the components $|i\rangle|\tilde{i}\rangle$, without losing the perfect correlation.

The algebraic structure of the collapse equation, eq. (10), is closely related to that of the master equation, eq. (7). Expansion of eq. (10) around the fixed point $\rho=P_{i}$ gives, with $\mathcal{L}\left[P_{i}\right] P_{i}=0$,

$$
\frac{\mathrm{d}}{\mathrm{d} s} \tilde{\rho}=2 \mathcal{L}\left[P_{i}\right] \tilde{\rho}-2 P_{i} \tilde{\rho} P_{i}-2\left(1-P_{i}\right) \tilde{\rho}\left(1-P_{i}\right)-2 \tilde{\rho} \operatorname{Tr}\left(\tilde{\rho} P_{i}\right)
$$

The term $\mathcal{L}\left[P_{i}\right] \tilde{\rho}=\mathcal{L}\left[P_{i}\right] \rho$ on the r.h.s. decouples $P_{i} \rho P_{i}$ from the rest of the density matrix by making the offdiagonal components $\left(P_{i} \rho P_{j}\right.$ and $\left.P_{j} \rho P_{i}\right)$ decay, but does not alter the diagonal components. The next two terms on the r.h.s. make the diagonal components of $\tilde{\rho}$ decay, leading the evolution to the fixed point. The last term on the r.h.s. is of higher order in $\tilde{\rho}$.

The Lindblad operators also satisfy the relation,

$$
\mathcal{L}[\rho] P_{i}+\mathcal{L}\left[P_{i}\right] \rho=\mathcal{L}[\tilde{\rho}] P_{i}=O\left(\tilde{\rho}^{2}\right)
$$

$\mathcal{L}\left[P_{i}\right] \rho$ is the influence of the apparatus pointer state on the system density matrix, while $\mathcal{L}[\rho] P_{i}$ is the influence of the system density matrix on the apparatus pointer state. For pure states, the collapse equation is just

$$
\frac{\mathrm{d}}{\mathrm{d} s} \rho=-2 \mathcal{L}[\rho] P_{i}
$$

These expressions suggest an inverse relationship between the processes of decoherence and collapse. Such an action-reaction relationship can follow from a conservation law. Initially, the combined system-apparatus state evolves unitarily, establishing perfect correlation and without any decoherence. The subsequent new description would be that during the measurement process, when $\mathcal{L}[\rho] P_{i}$ decoheres the apparatus pointer state $P_{i}$ (it cannot remain in superposition), there is an equal and opposite effect $-\mathcal{L}[\rho] P_{i}$ on the system density matrix $\rho$, resulting in the state collapse.

\section{The qubit case and some tests}

All the previous results can be expressed in a considerably simpler form in case of the smallest quantum system, i.e. the two-dimensional qubit with $|0\rangle$ and $|1\rangle$ as the measurement basis vectors. Evolution of the density matrix during the measurement, eqs (18) and (17), is given by

$$
\begin{aligned}
& \frac{\mathrm{d}}{\mathrm{d} s} \rho_{00}(s)=2\left(w_{0}-w_{1}\right) \rho_{00}(s) \rho_{11}(s), \\
& \rho_{01}(s)=\rho_{01}(0)\left[\frac{\rho_{00}(s) \rho_{11}(s)}{\rho_{00}(0) \rho_{11}(0)}\right]^{1 / 2} .
\end{aligned}
$$

Selecting the trajectory weights as addition of Gaussian white noise to the instantaneous Born rule results in

$$
w_{0}-w_{1}=\rho_{00}-\rho_{11}+\xi \text {. }
$$

The same evolution has been obtained by Korotkov ${ }^{9}$, using the Bayesian measurement formalism for a qubit. Our analysis is more general and is applicable to any quantum system.

For a single transmon qubit undergoing Rabi oscillations, perturbations caused by its weak measurement have been observed and then successfully cancelled by a measurement result-dependent feedback shift of the Rabi 


\section{SPECIAL SECTION: QUANTUM MEASUREMENTS}

frequency ${ }^{10}$, all consistent with the description provided by eqs (23-25). We have verified the same behaviour for two qubit systems using numerical simulations based on eqs (18) and (17), and trajectory weights chosen analogous to eq. (25), e.g. perturbations due to weak measurements of $J_{z} \otimes J_{z}$ on a Bell state undergoing joint Rabi oscillations can be cancelled by a measurement resultdependent Rabi frequency shift. We could not make this cancellation strategy work, however, with simultaneous measurement of more than one commuting operators and independent shifts of individual Rabi frequencies.

\section{Open questions}

Our proposed collapse equation, eq. (10), is quadratically nonlinear. Nonlinear Schrödinger evolution is inappropriate in quantum mechanics, because it violates the well-established superposition principle. Nonlinear superoperator evolution for the density matrix is also avoided in quantum mechanics, because it conflicts with the probability interpretation for mixtures of density matrices. Nevertheless, nonlinear quantum evolutions need not be unphysical and have been invoked in attempts to solve the measurement problem ${ }^{12,13}$. Equation (10) can be a valuable intermediate step in such attempts to interpret collapse as a consequence of some unknown underlying dynamics. It is definitely worth keeping in mind that nonabelian gauge theories and general relativity are examples of well-established theories with quadratic nonlinearities in their dynamical equations.

Irrespective of the underlying dynamics that may lead to the collapse equation, eq. (10), it is worthwhile to test it at its face value. It readily produces an eigenstate of the measured observable as the measurement outcome, but predictions of its ensemble version, eq. (14), are not stochastic and do not reproduce the Born rule. So to judge its validity, it is imperative to ask the question: Do quantum systems exhibit this alternate evolution, and if so under what conditions? Experimental tests would require determination of the density matrix evolution, in the presence of weak measurements and with highly suppressed decoherence effects. Such tests are now technologically feasible. It is indeed possible to generalize and extend the Bayesian measurement formalism tests for a single qubit by Vijay et al. ${ }^{10}$ and Murch et al. ${ }^{11}$, to larger quantum systems. They would clarify what trajectory weights $w_{i}$ (including stochastic noise) are appropriate for describing ensemble dynamics of weak measurements.
Finally, it is useful to note that, if the fixed point collapse dynamics of eq. (10) can be realized in practice, it would provide an unusual strategy for quantum error correction. After encoding the logical Hilbert space as a suitable subspace of the physical Hilbert space, one only has to perform measurements in an eigenbasis that separates the logical subspace and the error subspace as orthogonal projections. The state would then return towards the logical subspace as long as its projection on the logical subspace is larger than that on the error subspace, even if no feedback operations based on the measurement results are carried out.

1. Lindblad, G., On the generators of quantum dynamical subgroups. Comm. Math. Phys., 1976, 48(2), 119-130.

2. Gorini, V., Kossakowski, A. and Sudarshan, E. C. G., Completely positive semigroups of $N$-level systems. J. Math. Phys., 1976, 17(5), 821-825.

3. Giulini, D., Joos, E., Kiefer, C., Kuptsch, J., Stamatescu, I.-O. and Zeh, H. D., Decoherence and the Appearance of a Classical World in Quantum Theory, Springer, 1996.

4. Wiseman, H. M. and Milburn, G. J., Quantum Measurement and Control, Cambridge University Press, 2010.

5. DeWitt, B. S. and Graham, N. (eds), The Many-Worlds Interpretation of Quantum Mechanics, Princeton University Press, 1973.

6. Bengaluru is the capital of the state of Karnataka in India. See http://www.karnatakatourism.org/

7. Aharonov, Y., Albert, D. Z. and Vaidman, L., How the result of a measurement of a component of the spin of a spin-1/2 particle can turn out to be 100. Phys. Rev. Lett., 1988, 60(14), 1351-1354.

8. Ghose, P., Measurement as spontaneous symmetry breaking, nonlocality and non-Boolean holism; arXiv:1008.2510[quant-ph].

9. Korotkov, A. N., Continuous quantum measurement of a double dot. Phys. Rev. B, 1999, 60(8), 5737-5742; Selective quantum evolution of a qubit state due to continuous measurement. Phys. Rev. B, 2001, 63(11), 115403-1-115403-15.

10. Vijay, R. et al., Stabilizing Rabi oscillations in a superconducting qubit using quantum feedback. Nature, 2012, 490(7418), 77-80.

11. Murch, K. W., Weber, S. J., Macklin, C. and Siddiqi, I., Observing single quantum trajectories of a superconducting quantum bit. $\mathrm{Na}$ ture, 2013, 502(7470), 211-214.

12. Gell-Mann, M. and Hartle, J. B., Classical equations for quantum systems. Phys. Rev. D, 1993, 47(8), 3345-3382.

13. Penrose, R., On gravity's role in quantum state reduction. Gen. Relativ. Gravit., 1996, 28(5), 581-600.

ACKNOWLEDGEMENTS. We thank Michel Devoret (Yale University, USA) and Rajamani Vijayaraghavan (TIFR, Mumbai) for helpful conversations. The slogan of the Karnataka Tourism Department was pointed out to us by Raymond Laflamme (IQC, Canada). P.K. is supported by a CSIR research fellowship.

doi: $10.18520 / \mathrm{v} 109 / \mathrm{i} 11 / 2017-2022$ 